\title{
Cooperative extremum seeking for a one-dimensional non-linear map using a two-robot formation
}

\author{
Anna Skobeleva, V. Ugrinovskii, Ian R. Petersen
}

\begin{abstract}
This paper presents a cooperative extremum seeking control scheme that uses a linear formation of two robots to find a maximum of an unknown one-dimensional nonlinear map. A consensus control algorithm is used to drive the centre of the formation and an integral feedback control loop is proposed to drive the gradient of an unknown field function to zero. Simulation results are provided to evaluate the performance of the proposed method.
\end{abstract}

\section{INTRODUCTION}

Extremum seeking (ES) control naturally has found its application in the design of source seeking algorithms for autonomous mobile robots, where the aim is to find a source that emits a signal of interest and the strength of the signal is a function of distance from the source. In this type of problem, the field is unknown and only measurements of the field value at current robots' locations are available.

Various ES control techniques are used in the literature for coordination of a single and multiple autonomous robots in source seeking applications. For the single agent case the auxiliary movements are needed to estimate the gradient of the field, while in multi agent schemes the gradient estimation is performed using cooperative field sensing and information sharing. The authors of [2] propose an algorithm for a group of double integrators. Assuming that the robots are able to keep a record of previous measurements, the authors combine gradient projection on the vehicle's trajectory with a spring inter-vehicle potential function to produce a control input for each robot. In the subsequent work [15], the algorithm is modified for a group of robots moving in a formation that follows a virtual leader; gradient value at the leader's location is estimated by combining the field values across all platforms. A gradient climbing algorithm is presented in [3], where the local field gradient and Hessian matrices are estimated by a finite-difference method and the Newton's optimization method is used to determine the next reference position for the formation leader.

Randomized optimization based ES algorithms for particle swarming and bacteria foraging are presented in [11],[18]. A hybrid ES controller for a single integrator is described by [13]. The vehicle first travels in discrete steps along the

Anna Skobeleva and Valeri Ugrinovskii are with School of Engineering and Information Technology, University of New South Wales, Canberra, Australia. Ian Petersen is with College of Engineering and Computer Science, Australian National University, Canberra, Australia. anna.skobeleva@student.adfa. edu. au

This research was supported by the Australian Research Council under grant DP160101121 straight line until the minimum in the current direction is found and the Recursive Smith-Powell Algorithm is then used to choose a new search direction.

Sliding mode control is used for ES with a single unicycle in [12], where the angular velocity is being tuned while the linear velocity is constant. Another example of a sliding mode controller for a unicycle like vehicle is presented in [8] where the robot aims to track a moving target. Sliding mode algorithms do not require high computational capabilities, are easy to implement and has been showed to be robust to measurement noise. However, the main disadvantage is that the controller switches between two extreme control points causing the so-called "chattering effect" that wears out the actuators.

A perturbation based ES control (PESC) method which emplolys a dither signal to estimate the gradient of an unknown function is introduced in [1]. An extensive research in PESC was conducted by Krstic and co-workers. In [20], [19], [7] algorithms for agents with single and double integrator and unicycle dynamics are discussed, where either angular or forward velocity is tuned using PESC. One of the last works on the stochastic PESC is presented in [10], where the authors use white noise as the perturbation signal and both forward and angular velocities are being tuned.

The authors of [17] propose a switching ES control scheme, where single integrator agents are carrying out individual exploration approximating field gradient using time differences and switching to cooperative exploration based on signal to noise ratio. Approach is tested a filed with circular level sets and shows to be robust to measurement noise. A dither-free approach to ES control is proposed in [9] that uses history data of the field measurements to estimate its gradient by the first-order least squares fit and the optimizer is just an integrator with an adaptation gain.

The series of works [14], [5], [4], [6] present an ES approach with a circular formation of agents, where the field gradient is estimated as an average of the measurements across all agents weighted by a displacement of the corresponding agent from the formation centre and the estimate is used as a reference velocity value for the formation centre, then a consensus control algorithm for the formation control. Stability is analyzed for circular and elliptical level sets. While [14] assumes all-to-all 
communication, [5] only considers communication between neighboring agents and additional consensus algorithm is used to agree on estimated gradient direction. In [4] the authors keep gradient estimation and formation control the same but propose gradient-ascent algorithm to drive the formation centre. An extension to the problem of moving target tracking is provided in [6].

In this paper we propose an ES control scheme for a two robot formation that employs consensus control and negative integral feedback to drive a formation of two robots to a maximum of a one-dimensional field. Without making an assumption of circular or elliptical level sets of the unknown field we show that for a field function with a bounded second derivative, the formation successfully converges to a bounded region around maximum of the field and stops there.

In Section II the proposed control loop is described and its stability is analysed. Section III presents the proposed cooperative extremum seeking algorithm for a two-robot formation driven by a consensus algorithm. Section IV provides simulation results, and in Section $\mathrm{V}$ we give a conclusion and a perspective on future work.

\section{PROBLEM STATEMENT}

A. Stabilization to zero of a non-linear function using integral feedback control

Consider a problem where we need to control an input $u$ so that a non-linear system output $y=f(u)$ is driven to zero using a negative integral feedback. The problem is shown in Figure 1, where $u_{0}$ is a zero of the function $f(u)$ and $K$ is a feedback gain, that is a design parameter. We want our control to guarantee that as $t \rightarrow \infty, u \rightarrow u_{0}$ and thus $y(t) \rightarrow 0$.

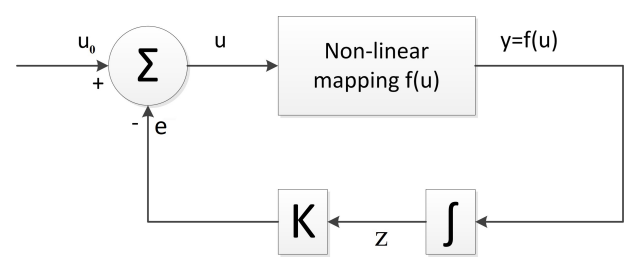

Fig. 1. The proposed feeback loop.

To analyze stability of the system in Figure 1, let $z$ denote the output of the integrator. Then we can write:

$$
\begin{aligned}
\dot{z}=y=f(u) \text { and } u & =u_{0}-K z, \text { that gives us: } \\
\dot{z} & =f\left(u_{0}-K z\right),
\end{aligned}
$$

Linearization of equation (1) using the first order Taylor expansion around $z=0$ gives us:

$$
\dot{z} \approx f\left(u_{0}\right)+f^{\prime}\left(u_{0}\right)\left(u_{0}-K z-u_{0}\right)=-K \alpha z
$$

where $\alpha=f^{\prime}\left(u_{0}\right)$. The solution of the equation (2) is $z(t)=$ $e^{-K \alpha t} z(0)$. Hence,

$$
y(t)=-K \alpha e^{-K \alpha t} z(0)
$$

From equation (3) we can see that $y(t) \rightarrow 0$ as $t \rightarrow \infty$, given that $K \neq 0, K \alpha>0$ and the initial condition $z(0)=$ $\frac{u_{0}-u(0)}{K}$ is sufficiently small for linearization errors to be negligible.

\section{B. Extention to the Extremum Seeking Control}

Inspired by the technique described in the previous section, we extend the proposed method to the extremum seeking control problem for a group of mobile robots. Now we think of the non-linear map whose zero is to be found as the gradient of a one-dimensional field of interest and of an input $u$ as a location of the robot formation centre.

Consider a linear formation of two mobile robots with single integrator kinematics:

$$
\dot{x}=v
$$

where $x$ and $v$ are the robot's position and linear velocity respectively. The problem is depicted in figure 2. The two robots (marked as 1 and 2) are moving along the $X$-axis, taking measurements of the function $f(x)$ along the way. Our goal is to drive the centre of the formation (marked with the yellow star) to a position $x_{\max }$ that corresponds to the maximum of the function.

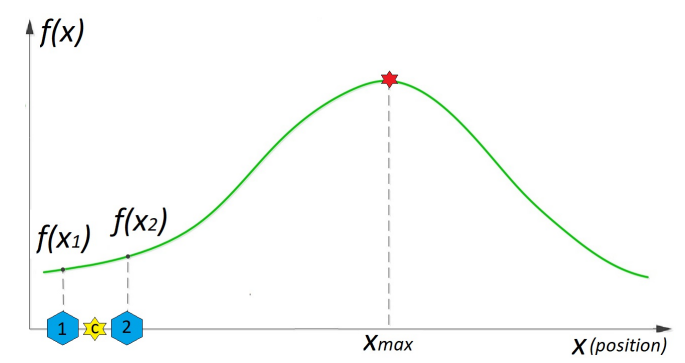

Fig. 2. The two robot setup for one-dimentional extremum seeking.

\section{EXTREMUM SEEKING SCHEME WITH CONSENSUS CONTROL}

To adopt the technique from the previous section we add the robots' kinematics and velocity control in the loop.

\section{A. Consensus control setup}

We propose to use consensus control to drive the formation centre to the extremum and a simple proportional control for robots' velocity inputs. The information state for the consensus control algorithm is the location of the formation centre. We start with the dynamic consensus tracking algorithm involving $n$ agents from [16], where information state of the $i^{t h}$ robot evolves as:

$$
\dot{x}_{i}(t)=-\sum_{j=1}^{n} a_{i j}(t)\left[x_{i}(t)-x_{j}(t)\right]+e(t),
$$


where $a_{i j}(t)$ is an entry of the adjacency matrix, $x_{i}(t)$ is the location of the formation centre as perceived by the $i^{\text {th }}$ robot and $e(t)$ is a driving signal generated by the feedback loop, that is an approximation of the gradient of the field and for a single integrator dynamics is equivalent to a reference velocity direction. The consensus algorithm in (5) aim to ensure that all agents track the formation centre. To derive control inputs for each robot, we add desired state deviations into the consensus algorithm, so we modify (5) as follows:

$$
\dot{x}_{i}(t)=-\sum_{j=1}^{n} a_{i j}(t)\left[x_{i}(t)-x_{j}(t)-\left(\delta_{i}-\delta_{j}\right)\right]+e(t),
$$

where $\delta_{1,2}=\mp h$ are desired distances of the robots from the formation centre. The proposed control loop diagram is presented in Figure 3. Now in the system in Figure 3 and equation (6) $x_{1}$ and $x_{2}$ are positions of the robots, and the $\pm 2 h$ offset is introduced to ensure the robots maintain the desired formation which is to keep them $2 h$ meters apart. We also introduce two adaptation gains $K_{p}$ in the velocity control loop and $K$ in the feedback loop to be able to regulate formation velocity and strength of the driving signal $e(t)$ in the consensus algorithm.

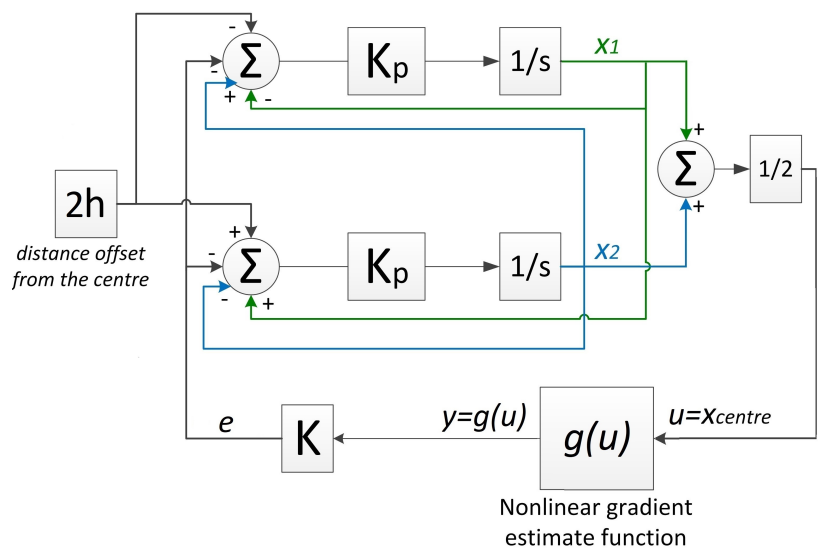

Fig. 3. Basic loop extension to consensus control.

\section{B. Convergence analysis}

To analyze the stability of the proposed control loop we write system equations expanding (6) for $x_{1}$ and $x_{2}$, with $\delta_{1,2}=\mp h$ and adjacency matrix $A=\left[\begin{array}{ll}0 & 1 \\ 1 & 0\end{array}\right]$, and adding proportional gain $K_{p}$ into the velocity control loop.

$$
\begin{gathered}
\dot{x_{1}}=K_{p}\left(-e-x_{1}+x_{2}-2 h\right), \\
\dot{x_{2}}=K_{p}\left(-e-x_{2}+x_{1}+2 h\right) \\
u=\left[\begin{array}{ll}
\frac{1}{2} & \frac{1}{2}
\end{array}\right]\left[\begin{array}{l}
x_{1} \\
x_{2}
\end{array}\right]
\end{gathered}
$$

where the input $e(t)=K g(u)$ is coming from the feedback loop and the output is $u(t)=x_{\text {centre }}(t)$. From (8) and (7) we can write:

$$
\dot{u}=\frac{1}{2}\left(\dot{x}_{1}+\dot{x_{2}}\right)=-K_{p} e
$$

whose transfer function $H(s)$ is given in (10) below.

$$
H(s)=-\frac{K_{p}}{s}
$$

After these transformations, the system in Figure 3 reduces to the system in Figure 4, since the dynamics of $x_{2}-x_{1}$ are decoupled from the dynamics of the formation centre. We can see that the equivalent transfer function of the consensus control already contains an integrator so we do not need to include one in the feedback loop. The equivalent block diagram is the same as the basic control loop presented in Figure 1 and is stable as long as $K<0$ so that $K \alpha>0$, as we have shown in Section II.A. Here, $\alpha=f^{\prime \prime}\left(x_{\text {maximum }}\right)<0$ for a maximum of the field function.

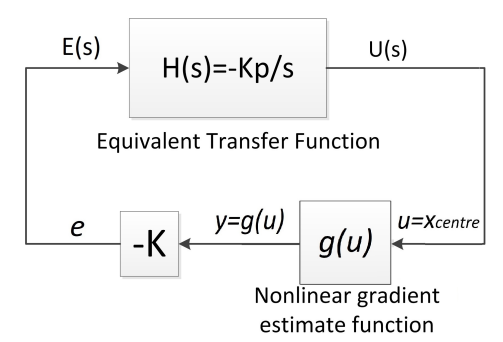

Fig. 4. Equivalent diagram the the consensus loop.

\section{Linearized approximated gradient algorithm}

A gradient approximation must be used to enable the implementation the proposed control scheme as we do not have the possibility to measure the gradient value directly. For a linear formation of two robots the gradient value at the centre of the formation is approximated using the finite difference formula:

$$
g(u)=\frac{f\left(x_{2}\right)-f\left(x_{1}\right)}{\left(x_{2}-x_{1}\right)}
$$

where $f\left(x_{1}\right), f\left(x_{2}\right)$ are measurements of the field taken by the robots at their corresponding locations. Now using (11) for the diagram in Figure 3 we can write:

$$
e=K \frac{f\left(x_{2}\right)-f\left(x_{1}\right)}{\left(x_{2}-x_{1}\right)}
$$

$K$ is a constant. When $x_{1}=x_{2}$, we assume by continuity that $\frac{f\left(x_{2}\right)-f\left(x_{1}\right)}{\left(x_{2}-x_{1}\right)}=f^{\prime}\left(x_{1}\right)=f^{\prime}\left(x_{2}\right)$.

Let $f$ be a function $\mathrm{R}^{1} \rightarrow \mathrm{R}^{1}$ which has the following properties:

(i) The function $f$ and its derivatives $f^{\prime}, f^{\prime \prime}$ are continuous;

(ii) The function $f$ has a single maximum, i.e., there is one and only one point $x^{*}$ such that $f^{\prime}\left(x^{*}\right)=0$.

(iii) The second derivative $f^{\prime \prime}(x)$ is uniformly bounded on $\mathrm{R}^{1},\left|f^{\prime \prime}(x)\right| \leq M$ for some $M>0$.

(iv) At the maximum point $x^{*}, f^{\prime \prime}\left(x^{*}\right)<0$.

(v) For arbitrary $x_{1}, x_{2}$,

$$
\left|f^{\prime \prime}\left(x_{1}\right)-f^{\prime \prime}\left(x_{2}\right)\right| \leq L .
$$


Let $\delta=x_{2}-x_{1}-2 h$, then it follows from (7) that

$$
\dot{\delta}=-2 K_{p} \delta, \quad \delta(0)=x_{2}(0)-x_{1}(0)-2 h .
$$

Thus, $\delta(t)=e^{-2 K_{p} t} \delta(0)$, and if $K_{p}>0$, then $\lim _{t \rightarrow \infty} \delta(t)=0$.

Next, we observe from (9) and (12) that

$$
\dot{u}=-K K_{p} \frac{f\left(x_{2}\right)-f\left(x_{1}\right)}{x_{2}-x_{1}}
$$

Let us consider the expression on the right hand side. Expand $f\left(x_{1}\right), f\left(x_{2}\right)$ in the vicinity of $u=\frac{x_{1}+x_{2}}{2}$ using the Taylor expansion formula with the Lagrange reminder,

$$
\begin{aligned}
& f\left(x_{1}\right)=f(u)+f^{\prime}(u) \frac{x_{1}-x_{2}}{2}+\frac{1}{2} f^{\prime \prime}\left(\xi_{1}\right) \frac{\left(x_{1}-x_{2}\right)^{2}}{4}, \\
& f\left(x_{2}\right)=f(u)+f^{\prime}(u) \frac{x_{2}-x_{1}}{2}+\frac{1}{2} f^{\prime \prime}\left(\xi_{2}\right) \frac{\left(x_{2}-x_{1}\right)^{2}}{4},
\end{aligned}
$$

where $\xi_{1}, \xi_{2}$ are some points between $x_{1}$ and $u$ and between $x_{2}$ and $u$, respectively. Then from (15),

$$
\dot{u}=-K K_{p} f^{\prime}(u)-\frac{K K_{p}}{8}\left(f^{\prime \prime}\left(\xi_{2}\right)-f^{\prime \prime}\left(\xi_{1}\right)\right)(\delta+2 h) .
$$

Using the notation $z=u-x^{*}$, the Taylor expansion of $f^{\prime}(u)$ can be written as:

$$
\begin{aligned}
f^{\prime}(u) & =f^{\prime}\left(x^{*}\right)+f^{\prime \prime}\left(\xi_{3}\right) z=f^{\prime}\left(x^{*}\right)+f^{\prime \prime}\left(x^{*}\right) z \\
& +\left(f^{\prime \prime}\left(\xi_{3}\right)-f^{\prime \prime}\left(x^{*}\right)\right) z
\end{aligned}
$$

where $\xi_{3}$ is a point between $u$ and $x^{*}$. Note that this is the exact identity. Recall that $f^{\prime}\left(x^{*}\right)=0$ and substitute into (17)

$$
\begin{aligned}
\dot{z}= & -K K_{p} f^{\prime \prime}\left(x^{*}\right) z-K K_{p}\left(f^{\prime \prime}\left(\xi_{3}\right)-f^{\prime \prime}\left(x^{*}\right)\right) z \\
& -\gamma(t)(\delta(t)+2 h)=-K K_{p} f^{\prime \prime}\left(x^{*}\right) z-\beta(t) z \\
& -\gamma(t) \delta(t)-2 \gamma(t) h,
\end{aligned}
$$

where

$$
\begin{aligned}
& \beta(t)=K K_{p}\left(f^{\prime \prime}\left(\xi_{3}(t)\right)-f^{\prime \prime}\left(x^{*}\right)\right), \\
& \gamma(t)=\frac{K K_{p}}{8}\left(f^{\prime \prime}\left(\xi_{2}(t)\right)-f^{\prime \prime}\left(\xi_{1}(t)\right)\right) .
\end{aligned}
$$

Now combine (18) and (14). Let $\chi=\left(\begin{array}{l}\delta \\ z\end{array}\right)$, then

$$
\begin{gathered}
\dot{\chi}=\underbrace{\left[\begin{array}{cc}
-2 K_{p} & 0 \\
0 & -K K_{p} f^{\prime \prime}\left(x^{*}\right)
\end{array}\right]}_{A} \chi+\underbrace{\left[\begin{array}{c}
0 \\
-1
\end{array}\right]}_{B} \phi_{1}\left(t, \zeta_{1}\right) \\
+\left[\begin{array}{c}
0 \\
-1
\end{array}\right] \phi_{2}\left(t, \zeta_{2}\right)+\left[\begin{array}{c}
0 \\
-1
\end{array}\right] \phi_{3}(t) \\
\phi_{1}\left(t, \zeta_{1}\right)=\gamma(t) \zeta_{1} \quad \zeta_{1}=\delta=\underbrace{\left[\begin{array}{ll}
1 & 0
\end{array}\right]}_{=C_{1}} \chi, \\
\phi_{2}\left(t, \zeta_{2}\right)=\beta(t) \zeta_{2} \quad \zeta_{2}=z=\underbrace{\left[\begin{array}{ll}
0 & 1
\end{array}\right]}_{=C_{2}} \chi, \\
\phi_{3}(t)=2 \gamma(t) h .
\end{gathered}
$$

According to $(\mathrm{v})$,

$\left|\phi_{1}\left(t, \zeta_{1}\right)\right|<\alpha_{1}\left|\zeta_{1}\right|,\left|\phi_{2}\left(t, \zeta_{2}\right)\right|<\alpha_{2}\left|\zeta_{2}\right|, \quad\left|\phi_{3}(t)\right|<2 \alpha_{1} h$,

where $\alpha_{1}=\frac{|K| K_{p} L}{8}, \alpha_{2}=|K| K_{p} L$. That is the system (20) can be considered as an uncertain system with two 'norm-bounded' uncertainty inputs $\phi_{1}, \phi_{2}$ subject to the constraints (21), and a bounded disturbance $\phi_{3}$.

Asymptotic properties of the system (20) can be established using the S-procedure method. Choose $V(\chi)=\chi^{\prime} P \chi$ as a candidate Lyapunov function. Let $\tau_{1}, \tau_{2}>0$ and $\varepsilon>0$ be three constants. Consider

$$
\begin{aligned}
& \dot{V}-\tau_{1}\left(\left|\phi_{1}\right|^{2}-\alpha_{1}^{2}\left|\zeta_{1}\right|^{2}\right)-\tau_{2}\left(\left|\phi_{2}\right|^{2}-\alpha_{2}^{2}\left|\zeta_{2}\right|^{2}\right) \\
& =2 \chi P\left(A \chi+B \phi_{1}+B \phi_{2}+B \phi_{3}\right)-\tau_{1}\left(\left|\phi_{1}\right|^{2}-\alpha_{1}^{2}\left|C_{1} \chi\right|^{2}\right) \\
& -\tau_{2}\left(\left|\phi_{2}\right|^{2}-\alpha_{2}^{2}\left|C_{2} \chi\right|^{2}\right)=\chi^{\prime}\left(P A+A^{\prime} P+\tau_{1} \alpha_{1}^{2} C_{1}^{\prime} C_{1}\right. \\
& \left.+\tau_{2} \alpha_{2}^{2} C_{2}^{\prime} C_{2}+\frac{1}{\tau_{1}} P B B^{\prime} P+\frac{1}{\tau_{2}} P B B^{\prime} P+\frac{1}{\varepsilon} P B B^{\prime} P\right) \chi \\
& -\tau_{1}\left|\phi_{1}-\frac{1}{\tau_{1}} B^{\prime} P \chi\right|^{2}-\tau_{2}\left|\phi_{2}-\frac{1}{\tau_{2}} B^{\prime} P \chi\right|^{2}+\varepsilon\left|\phi_{3}\right|^{2}
\end{aligned}
$$

Now suppose that for some $\lambda>0$, the Linear Matrix Inequality

$$
\left[\begin{array}{cccc}
P A+A^{\prime} P+\tau_{1} \alpha_{1}^{2} C_{1}^{\prime} C_{1} & P B & P B & P B \\
+\tau_{2} \alpha_{2}^{2} C_{2}^{\prime} C_{2}+\lambda P & & & \\
B^{\prime} P & -\tau_{1} & 0 & 0 \\
B^{\prime} P & 0 & -\tau_{2} & 0 \\
B^{\prime} P & 0 & 0 & -\varepsilon
\end{array}\right]<0
$$

admits a solution $P=P^{\prime}>0, \tau_{1}>0, \tau_{2}>0, \varepsilon>0$. Then for all $\phi_{1}, \phi_{2}$, and $\phi_{3}$

$$
\begin{aligned}
& \dot{V}+\lambda V<\tau_{1}\left(\left|\phi_{1}\right|^{2}-\alpha_{1}^{2}\left|C_{1} \chi\right|^{2}\right) \\
& +\tau_{2}\left(\left|\phi_{2}\right|^{2}-\alpha_{2}^{2}\left|C_{2} \chi\right|^{2}\right)+\varepsilon\left|\phi_{3}\right|^{2},
\end{aligned}
$$

and taking into account (21), we obtain that for all admissible $\phi_{1}, \phi_{2}, \phi_{3}$,

$$
\dot{V}+\lambda V<4 \varepsilon \alpha_{1}^{2} h^{2}=\frac{\varepsilon K_{p}^{2} K^{2} L^{2} h^{2}}{16},
$$

This implies that for any initial condition $\chi(0)$ the trajectories of the system (20), (21) are bounded as follows

$$
\begin{aligned}
|\delta(t)|^{2}+|z(t)|^{2} & \leq \frac{1}{\sigma_{\min }(P)} e^{-\lambda t}\left(|\delta(0)|^{2}+|z(0)|^{2}\right) \\
& +\frac{\varepsilon K_{p}^{2} K^{2} L^{2} h^{2}}{16 \lambda \sigma_{\min }(P)} .
\end{aligned}
$$

Note that $\delta(t) \rightarrow 0$, therefore we have the following theorem as our main result.

Theorem 1 Suppose the function $f$ satisfies conditions (i)(v). Also, suppose that there exist $K, K_{p}$ and $\lambda>0$ such that the LMI (23) is feasible; i.e., there exists a matrix $P>0$ and constants $\tau_{1}>0, \tau_{2}>0, \epsilon>0$ such that (23) holds. Then the approximated gradient algorithm converges in the sense that $\lim _{t \rightarrow \infty}\left|x_{1}-x_{2}\right|=2 h$ and

$$
\limsup _{t \rightarrow \infty}\left|\frac{x_{1}+x_{2}}{2}-x^{*}\right| \leq \frac{\varepsilon K_{p}^{2} K^{2} L^{2} h^{2}}{16 \lambda \sigma_{\min }(P)}
$$




\section{Simulation RESUlTS}

To evaluate the performance of the scheme proposed in the previous section, we use the Matlab Simulink environment. The nonlinear field function for our simulations is of the form:

$$
\begin{aligned}
f(x)= & 40 \sin (2 \pi * 0.002(x-100)) \\
& +10 \sin \left(2 \pi * 0.0055(x-100)+3 \frac{\pi}{4}\right)
\end{aligned}
$$

where $x$ is in meters. On the interval $x \in[0,400]$ the function has a single maximum point at $x=u_{0}=247.178$ and $\ddot{f}\left(u_{0}\right)<0$. A plot of the function and its gradient is shown in Figure 5.
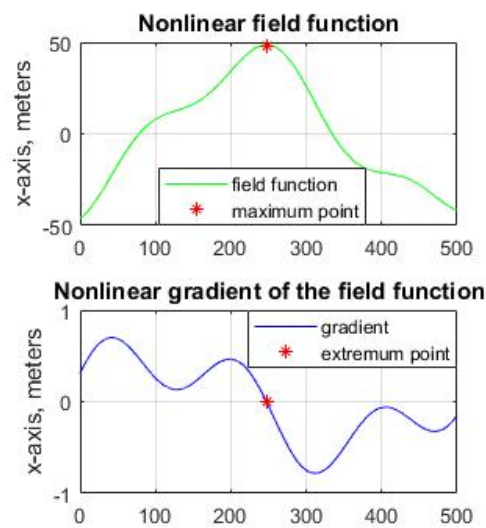

Fig. 5. Nonlinear function used for simulations.

Simulation results for two different initial locations are presented in Figures 6, 7. These simulations show that the robots converge to the desired formation (distance offset) and the formation converges to the neighborhood of the maximum point of the field and stops there.

For an implementation on a real hardware, we need to consider actuator saturation. Even though we did not conduct a stability analysis with saturation non-linearity in the loop, we carried out simulations with the robots' maximum velocity capped at $1.5 \mathrm{~m} / \mathrm{sec}$ and have shown that the formation still reaches the extremum and stops there. Simulation results are presented in Figure 8.

\section{CONCLUSION AND FUTURE WORK}

We have proposed a cooperative exremum seeking control scheme that uses consensus control and an integral feedback loop to drive a two-robot formation to the maximum of an unknown non-linear map. We have found that the consensus algorithm itself gives us an integrator so we do not need to include one in the feedback loop. We have analysed the stability of the proposed loop and demonstrated convergence to the neighborhood of the extremum point using simulations. The simulations have shown that the proposed scheme works well even when a velocity saturation is accounted
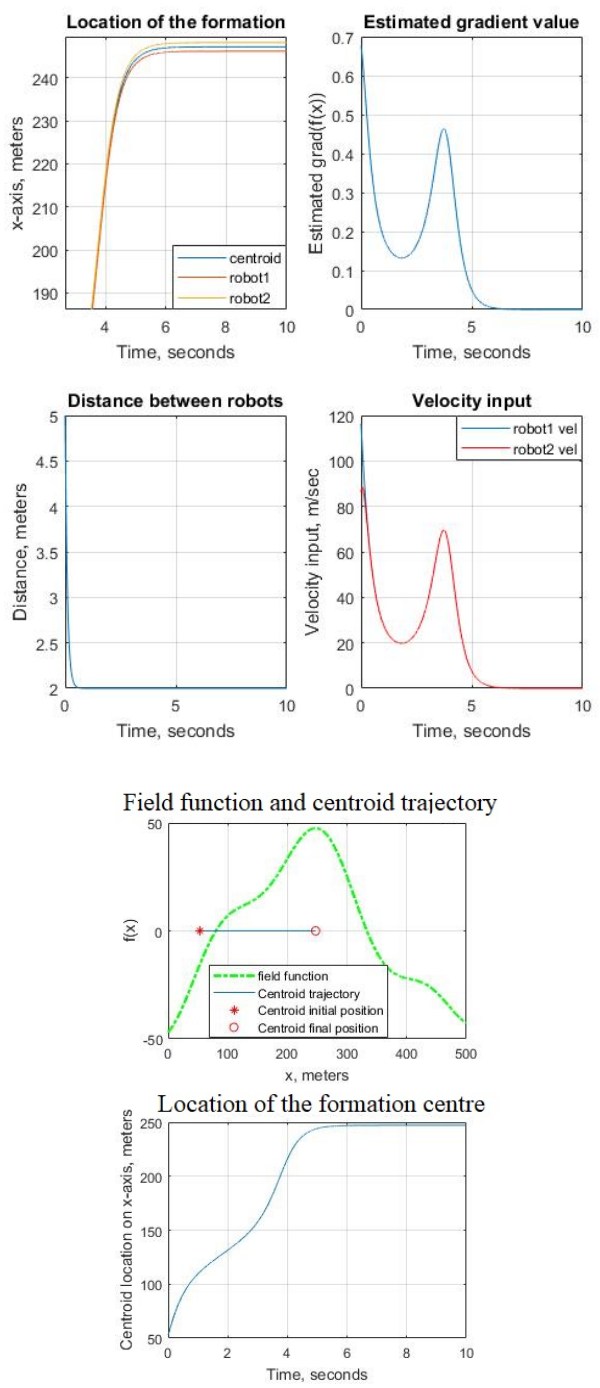

Fig. 6. Simulation results for initial robot locations $x_{1}=50$, $x_{2}=55$

for in the loop, that is a very important aspect for physical implementation.

In future work we will consider a nonlinear stability analysis that would allow us to determine what types of nonlinear field functions our control scheme can handle. We also are aiming to extend our work to handle two-dimensional extremum seeking on a plane, and use a more accurate gradient estimation technique for a formation of multiple (three or more) robots.

\section{REFERENCES}

[1] K. B. Ariyur and M. Krstic, Real-time optimization by extremumseeking control. John Wiley \& Sons, 2003.

[2] R. Bachmayer and N. E. Leonard, "Vehicle networks for gradient descent in a sampled environment," in Decision and Control, 2002, Proceedings of the 41st IEEE Conference on, vol. 1. IEEE, 2002, pp. 112-117.

[3] E. Biyık and M. Arcak, "Gradient climbing in formation via extremum seeking and passivity-based coordination rules," Asian Journal of Control, vol. 10, no. 2, pp. 201-211, 2008. 

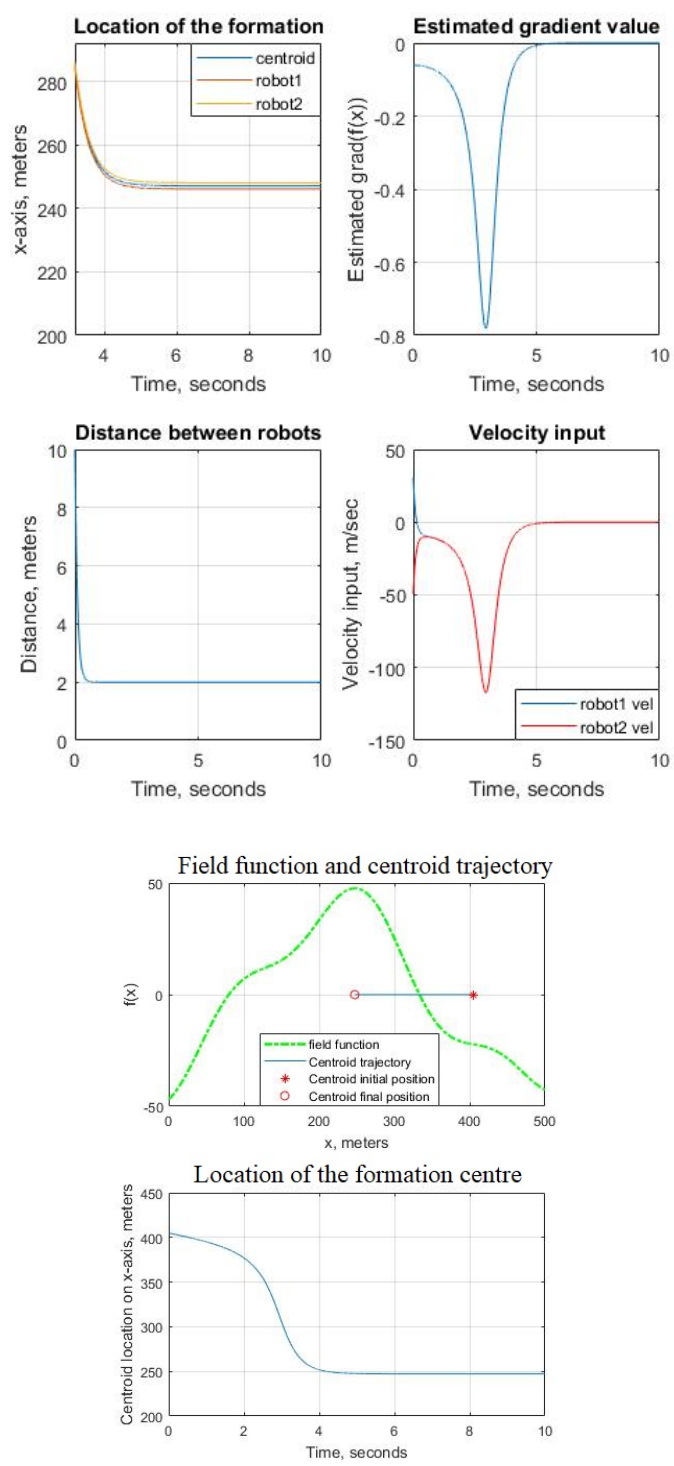

Fig. 7. Simulation results for initial robot locations $x_{1}=400$, $x_{2}=410$

[4] L. Brinón-Arranz and L. Schenato, "Consensus-based source-seeking with a circular formation of agents," European Control Conferenc e, pp. 2831-2836, 2013.

[5] L. Brinón-Arranz, A. Seuret, and C. Canudas-de Wit, "Collaborative estimation of gradient direction by a formation of auvs under communication constraints," in Decision and Control and European Control Conference (CDC-ECC), 2011 50th IEEE Conference on. IEEE, 2011, pp. 5583-5588.

[6] L. Briñón-Arranz, A. Seuret, and A. Pascoal, "Target tracking via a circular formation of unicycles," 2017.

[7] J. Cochran and M. Krstic, "Nonholonomic source seeking with tuning of angular velocity," Automatic Control, IEEE Transactions on, vol. 54, no. 4, pp. 717-731, 2009.

[8] L. Fu and U. Ozguner, "Sliding mode in constrained source tracking with non-holonomic vehicles," in Variable Structure Systems, 2008. VSS'08. International Workshop on. IEEE, 2008, pp. 30-34.

[9] B. Hunnekens, M. Haring, N. van de Wouw, and H. Nijmeijer, "A dither-free extremum-seeking control approach using 1st-order leastsquares fits for gradient estimation," in Decision and Control (CDC), 2014 IEEE 53rd Annual Conference on. IEEE, 2014, pp. 2679-2684.

[10] J. Lin, S. Song, K. You, and M. Krstic, "Stochastic source seeking
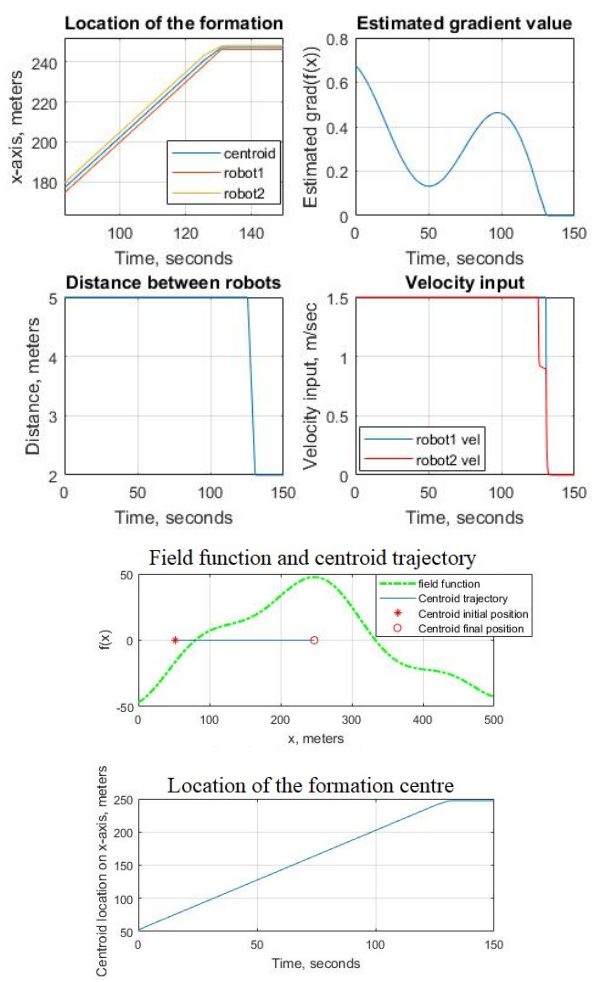

Fig. 8. Simulation results for the case of a velocity input with saturation.

with forward and angular velocity regulation," Automatica, vol. 83, pp. 378-386, 2017.

[11] H. Liu, H. Chen, and L. Kong, "Bacteria foraging optimization-based extremum seeking control," in Intelligent Computing and Intelligent Systems (ICIS), 2010 IEEE International Conference on, vol. 3. IEEE, 2010, pp. 110-115.

[12] A. S. Matveev, H. Teimoori, and A. V. Savkin, "Navigation of a unicycle-like mobile robot for environmental extremum seeking," Automatica, vol. 47, no. 1, pp. 85-91, 2011.

[13] C. G. Mayhew, R. G. Sanfelice, and A. R. Teel, "Robust sourceseeking hybrid controllers for autonomous vehicles," in American Control Conference, 2007. ACC'07. IEEE, 2007, pp. 1185-1190.

[14] B. J. Moore and C. Canudas-de Wit, "Source seeking via collaborative measurements by a circular formation of agents," in American Control Conference (ACC), 2010. IEEE, 2010, pp. 6417-6422.

[15] P. Ogren, E. Fiorelli, and N. E. Leonard, "Cooperative control of mobile sensor networks: Adaptive gradient climbing in a distributed environment," Automatic Control, IEEE Transactions on, vol. 49 , no. 8, pp. 1292-1302, 2004.

[16] W. Ren and R. Beard, Distributed consensus in multi-vehicle cooperative control: theory and applications. Springer Science \& Business Media, 2007.

[17] W. Wu and F. Zhang, "Robust cooperative exploration with a switching strategy," IEEE Transactions on Robotics, vol. 28, no. 4, pp. 828-839, 2012.

[18] S.-J. Yu, H. Chen, and L. Kong, "Particle swarm optimizationbased extremum seeking control," in Advanced Intelligent Computing Theories and Applications. Springer, 2010, pp. 185-196.

[19] C. Zhang, D. Arnold, N. Ghods, A. Siranosian, and M. Krstic, "Source seeking with non-holonomic unicycle without position measurement and with tuning of forward velocity," Systems \& control letters, vol. 56, no. 3, pp. 245-252, 2007.

[20] C. Zhang, A. Siranosian, and M. Krstić, "Extremum seeking for moderately unstable systems and for autonomous vehicle target tracking without position measurements," Automatica, vol. 43, no. 10, pp. 1832-1839, 2007. 